\title{
A Scanning Force Microscope Combined with a Scanning Electron Microscope for Multidimensional Data Analysis
}

\author{
Michel Troyon $\left({ }^{1}\right)$, Hei Ning Lei $\left({ }^{1}\right)$, Zhonghuai Wang $\left({ }^{1}\right)$ \\ and Guangyi Shang $\left({ }^{2}\right)$ \\ $\left({ }^{1}\right)$ Unité de Thermique et Analyse Physique $\left({ }^{*}\right)$, Laboratoire de Microscopies Électronique \\ et Tunnel, 21 rue Clément Ader, 51685 Reims Cedex 2, France \\ $\left({ }^{2}\right)$ STM Laboratory, Institute of Chemistry, Chinese Academy of Sciences, \\ Beijing 100080, China
}

(Received February 2, 1998; accepted February 13, 1998)

\begin{abstract}
PACS.07.79.-v - Scanning probe microscopes, components, and techniques
PACS.07.78.+s - Electron, positron, and ion microscopes, electron diffractometers, and related techniques
\end{abstract}

\begin{abstract}
A Scanning Force Microscope (SFM) intended for operation inside a Scanning Electron Microscope (SEM) is described. This combined instrument allows one to image a sample conventionally by SEM and to investigate by SFM the local topography as well as certain physical characteristics of the surface (friction, elasticity...). The combination of the two microscopes is very attractive because they complement each other in terms of depth of field, lateral and vertical resolution, field of view, speed and ability to image insulating surfaces. A multidimensional data space relative to the same area of a sample surface can be constructed, which should help to give new insights into the nature of materials.
\end{abstract}

\section{Introduction}

The Scanning Force Microscope (SFM) [3] has become an important surface analysis tool over the past few years. Its first function is, naturally, three-dimensional topographic analysis with high lateral and vertical resolution; it is thus usable as a profilometer at the nanometre scale. In other respects, it is able to give information about certain physical properties of the surface since the tip is sensitive to different types of forces or interactions. Tribological investigations can be envisaged by recording the effects of lateral frictional forces [2]. It is also possible to study the viscoelastic properties [17], adhesion [6], magnetic [14] or electrostatic [20] characteristics of different materials. SFM is used not only in surface physics but also in various fields such as semiconductor development and biology. In spite of its advantages, mainly high resolution and the ability to image non-conductive samples, the use of the SFM is limited by its small scan area and its slow scan speed.

The Scanning Electron Microscope (SEM) is also a surface analysis instrument. SEM generally has a lateral resolution of about $5 \mathrm{~nm}$, whereas a $\mathrm{SEM}$ equipped with a field emission

$\left({ }^{*}\right)$ E.A. 2061 
gun has a resolution of about $1 \mathrm{~nm}$. It must be pointed out that this resolution is obtained only in the case of very special test specimens capable of giving good contrast in the secondary electron imaging mode, gold particles on carbon for example. In most cases specimens do not present sufficient contrast to be seen with the resolution limit of the instrument. The SEM has some other very interesting characteristics, mainly its large depth of field and the possibility of imaging large areas of a few $\mathrm{mm}^{2}$ very quickly.

Scanning Tunnelling Microscopy (STM) is a technique closely related to SFM, with similar limitations (small scan area, slow scan speed). A few teams have tried to solve these limitations by combining the scanning tunnelling microscope with a SEM $[8,10,11,22,24]$. The STM/SEM hybrid instrument takes advantage of both techniques: the real-time image of the SEM makes it possible to view a wide field of the sample, choose a selected spot and then zoom onto that area with the STM. Nevertheless, our experience of STM/SEM combination showed us that the easy use of STM is restricted to very clean and well conducting specimens. Very often, imaging reliability was not good and tips broke because of contamination or poor conducting properties of the observed specimen.

The SFM being able to image insulators, this drawback is no more a problem with a combined SFM/SEM. SFM and SEM are therefore complementary techniques, the advantages of one compensating the drawbacks of the other. For the SEM user, besides improvements concerning the resolution (mainly the vertical resolution), a fundamental aspect is very attractive: SFM is able to provide multidimensional information from the surface (friction, adhesion, viscoelastic properties...).

A few attempts have been made recently to combine a SFM with a SEM $[7,12,19]$. The system we have developed is based on the widely used technique of the position-sensitive detection of a laser beam from the cantilever. A commercial SFM/SEM using this method is available but has not been described in the literature [21]. In the present article we describe the design of our combined SFM/SEM and its performance and we illustrate its potentialities with a few examples.

\section{SFM Description}

The basic principle of the SFM we have developed to be combined with a SEM is schematically explained in Figure 1. A $3 \mathrm{~mW}$ collimated laser diode (Melles-Griot) and a lens (focal length $25 \mathrm{~mm}$ ) are rigidly mounted on a flange, which can be orientated to centre the focused beam on the cantilever top. Two fixed mirrors reflect the beam on a four-quadrant photodetector (Advances Photonics Inc.), which can be moved in two perpendicular directions by means of a displacement mechanism. Topographic and lateral frictional force images can thus be simultaneously recorded. The piezoelectric ceramic (EBL\#3 from Staveley Sensors Inc.) is a tube of $6.5 \mathrm{~mm}$ diameter giving a scanning area of $8 \times 8 \mu \mathrm{m}^{2}$ and a $Z$ dynamic range of $\pm 1.6 \mu \mathrm{m}$.

The viewing plane of Figure 1 is $45^{\circ}$ tilted through with respect to the electron beam; that is, the specimen surface is also $45^{\circ}$ tilted, facing the secondary electron detector (Fig. 2). The specimen is placed at a working distance of $15 \mathrm{~mm}$ and a coarse positioner can move the specimen in the $X$ and $Y$ directions $( \pm 1,5 \mathrm{~mm}$ ) with two stepper motors ("inchworm" type from Burleigh). The laser diode, the two mirrors, the cantilever, and the photodetector are all mounted in a support (the SFM head) that can be separated from the SFM base so as to change the specimen. The SFM head is set on three mobile axes forming a tripod and maintained by two springs. Two of these axes are threaded and can be manually rotated for a coarse positioning of the cantilever with respect to the specimen. The third axis is the sliding 


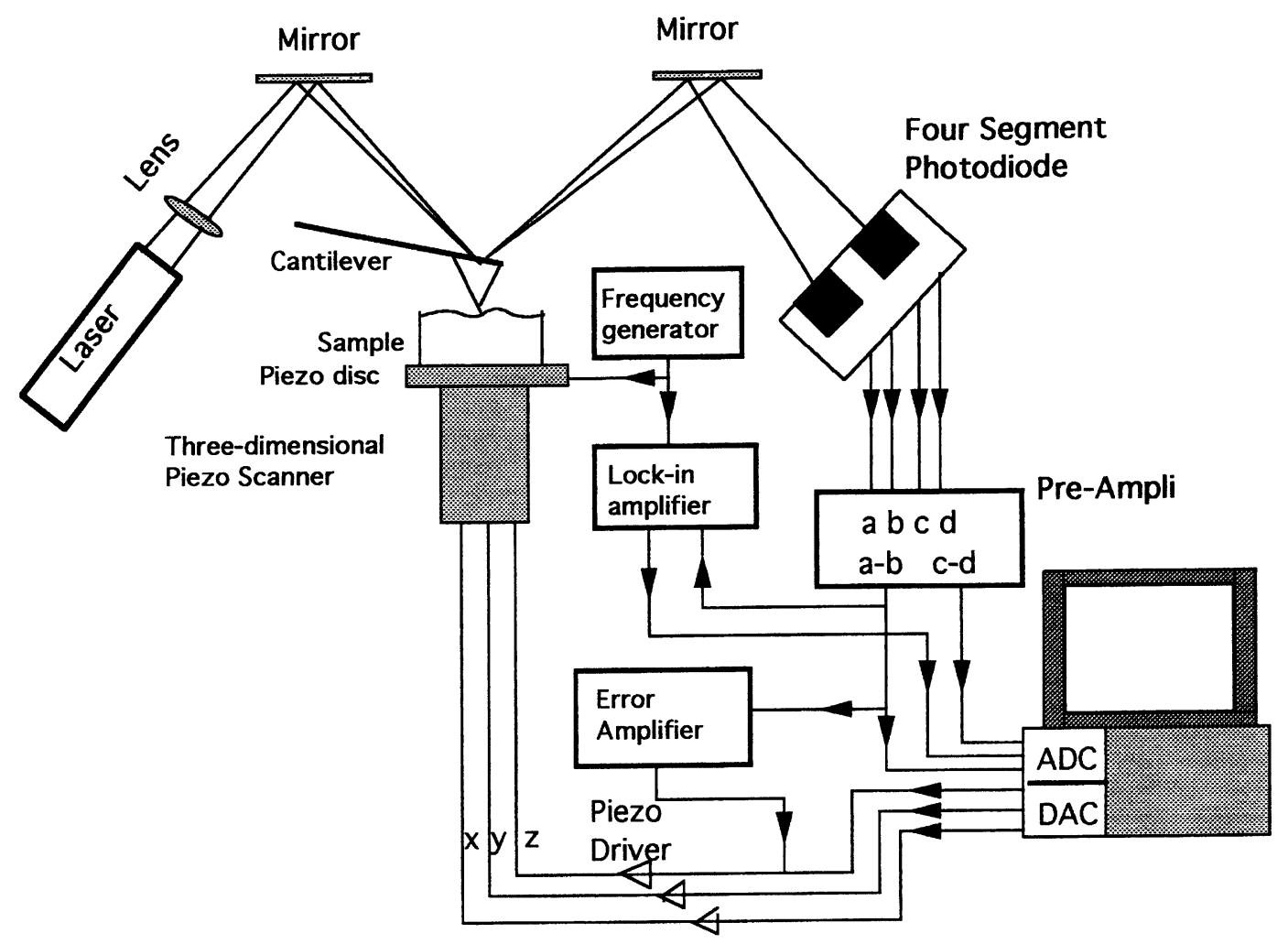

Fig. 1. - Schematic working principle of the SFM.

axis of a third inchworm motor allowing one to engage the tip in contact with the specimen. The three motors are of course controlled from outside the SEM when the SFM head is under vacuum. Figure 3 shows the SFM mounted on the object chamber stage of a commercial SEM (Philips SEM 515). We have deliberately chosen to connect the SFM rigidly to the specimen displacement stage of the SEM even though the problem of mechanical vibrations is crucial in near-field microscopy for obtaining the atomic resolution. There are two reasons for this choice. In our previous experience of combining an STM to a SEM [22] we chose to support the STM head on a stack of 5 plates separated by viton O-rings to reduce the mechanical vibrations. This was undoubtedly efficient for obtaining the STM atomic resolution but was detrimental to the SEM resolution because the specimen was not laterally stable with respect to the column axis. Furthermore, we think it is illusory and thus not useful to strive towards atomic resolution in a SEM under conventional vacuum because high resolution studies require that the sample surface be prepared under ultra-high vacuum conditions to avoid and preserve against any contamination.

The electronics are of analogue type and home-built on the basis of those developed by Radmacher [18]. The data acquisition, scan, display, processing and analysis are accomplished by a digital system (Macintosh Quadra 650) equipped with two interface cards (Macadios GW1-625 and GW1-DAC). The software, developed by Gaub's team, allows the simultaneous 


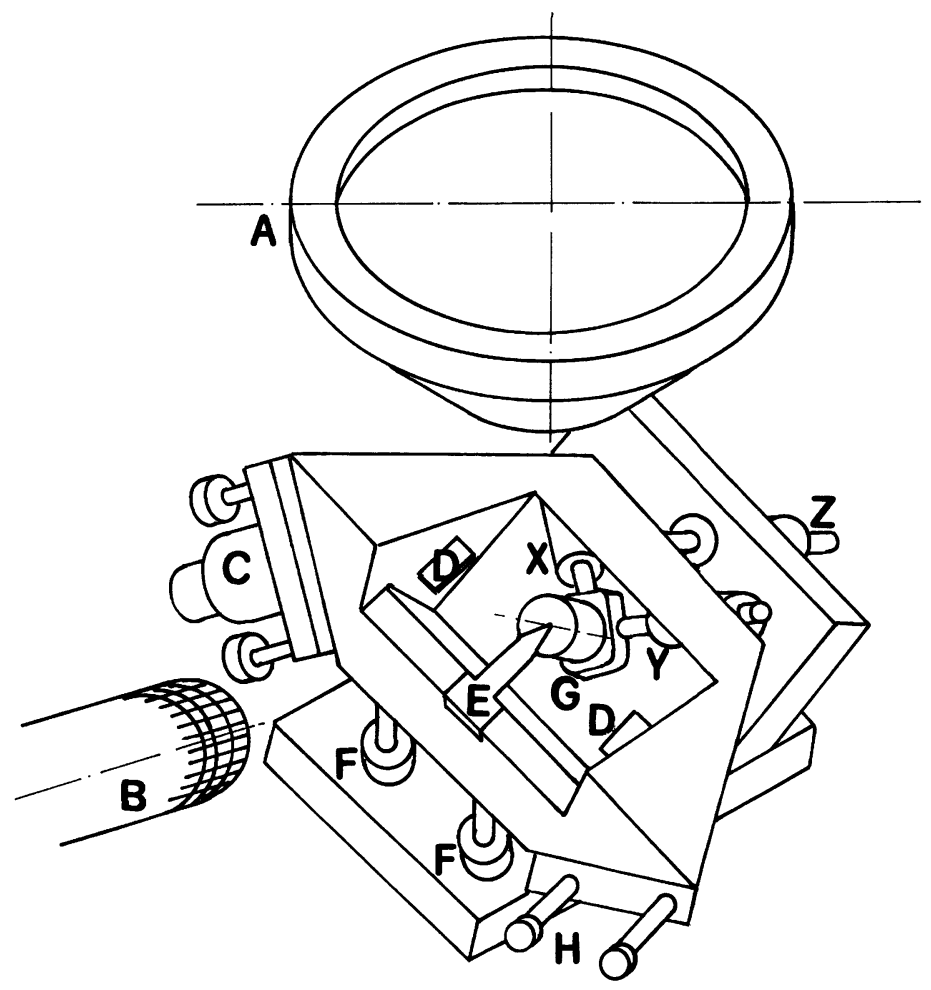

Fig. 2. - Schematic view of the SFM inside the SEM. (A) objective lens housing; (B) secondary electron detector; (C) laser diode; (D) mirrors; (E) cantilever support; (F) coarse approach screws; $(\mathrm{G})$ piezo tube and sample; $(\mathrm{H})$ photodetector adjustment screws; $(X, Y, Z)$ stepper motors.

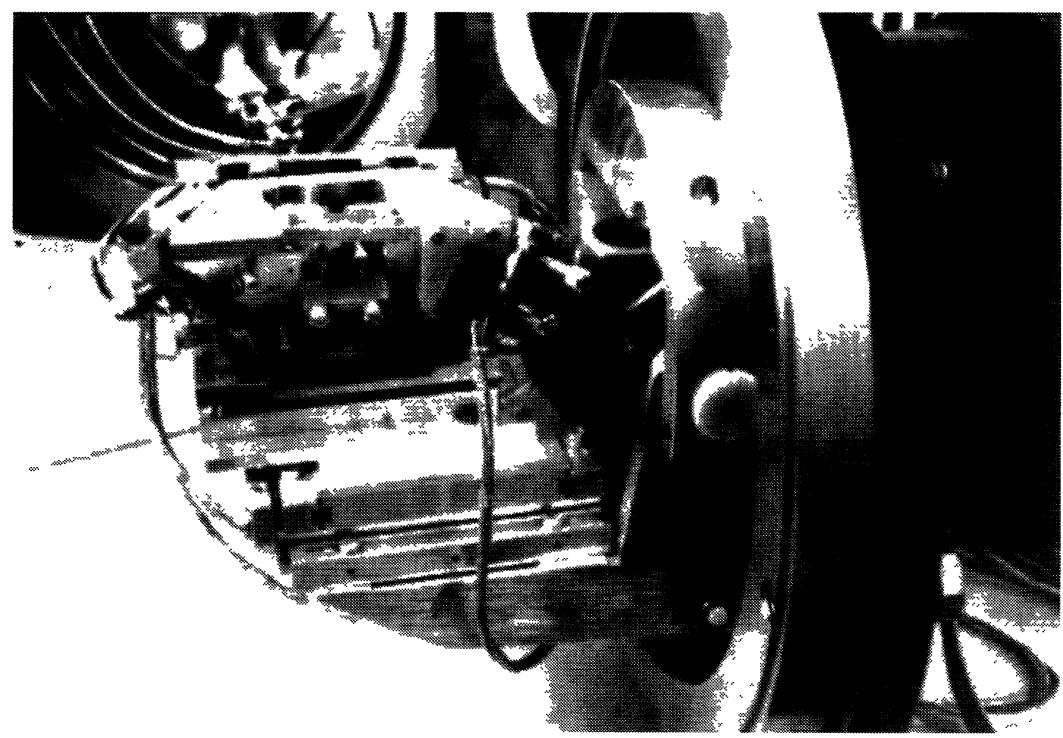

Fig. 3. - View of the SFM mounted inside the object chamber of SEM. 


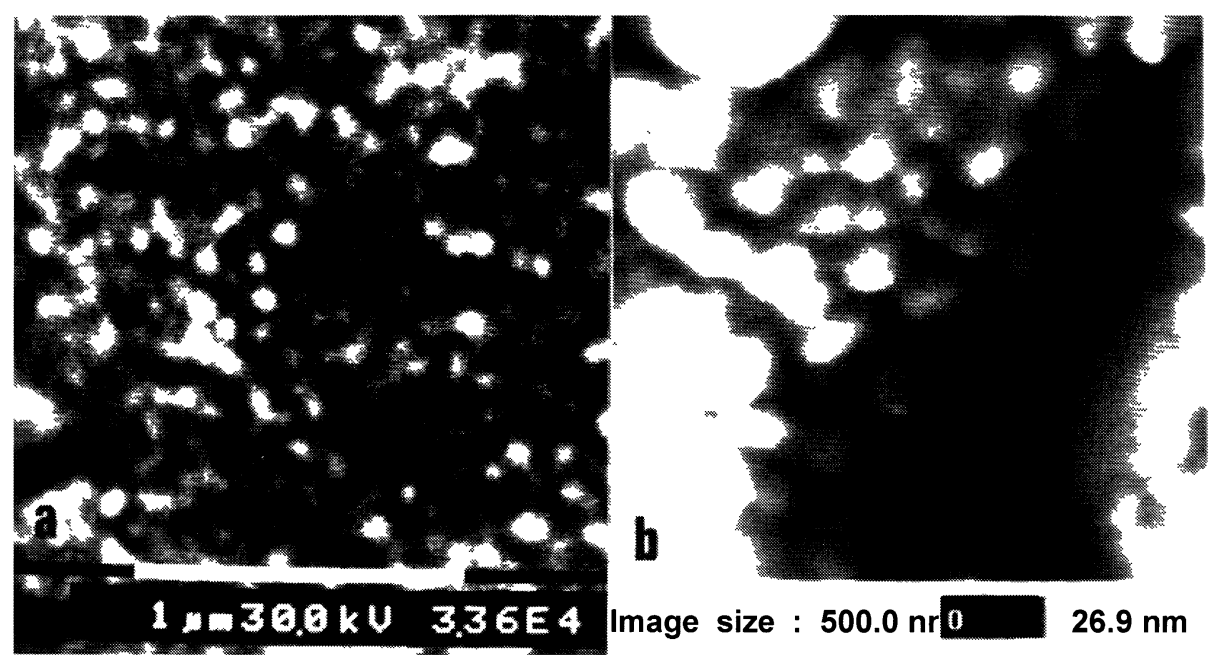

Fig. 4. - Images of gold particles evaporated on carbon obtained with the SFM/SEM combination. a) SEM image: working distance $=15.9 \mathrm{~mm}$; b) topographical SFM image.

acquisition of three types of different signals; forward and backward scans are always recorded, and so six images of $256 \times 256$ pixels are obtained.

\section{Results and Discussion}

The resolutions of the individual parts of the combined instrument have first been evaluated. Figure 4 shows the images of a classical test object regularly used to measure the SEM resolution (gold particles evaporated on carbon). Figure 4a is the SEM image taken at $30 \mathrm{kV}$. The resolution is evaluated to be of the order of $20 \mathrm{~nm}$, which is the normal image quality of our SEM for a working distance of $15.9 \mathrm{~mm}$; the presence of the SFM does not impair the SEM resolution. Figure $4 \mathrm{~b}$ is the SFM image of these gold particles. Line profiling indicates that these are $2-3 \mathrm{~nm}$ high, which is a lower limit because the tip is too broad to reach into the space between granules. Nevertheless, the resolution is much better than that of the SEM. It is difficult to quantify it since gold particles do not present fine structures at their surface but they are clearly distinguishable whereas some of these are only faintly visible in the SEM image. A STM rigidly connected to the specimen stage of a SEM has already been proved to be efficient for a good resolution [1]. Our own tests show also that this solution is a good one for a SFM/SEM combination. On the other hand, to verify the performance of our SFM, we tested it on an antivibration table in air; atomic resolution could be obtained on a mica sample.

The following example is presented to illustrate the interest of the combined SFM/SEM instrument but also to demonstrate the complementarity of the two techniques. Figure 5 shows that it is very easy to localise a particular spot of interest in a sample with the SEM, then to move the specimen under the probing tip and to zoom and reveal details by SFM that SEM is not able to show. Figure $5 \mathrm{a}$ is the SEM image of a two-dimensional grating made by electron microlithography. It consists of circular gold studs on a silicon substrate with a diameter of $200 \mathrm{~nm}$ and a periodicity of $400 \mathrm{~nm}$. The top of the cantilever can also be seen in this image. The tip used is a conventional $\mathrm{Si}_{3} \mathrm{~N}_{4}$ tip the radius of which is approximately 

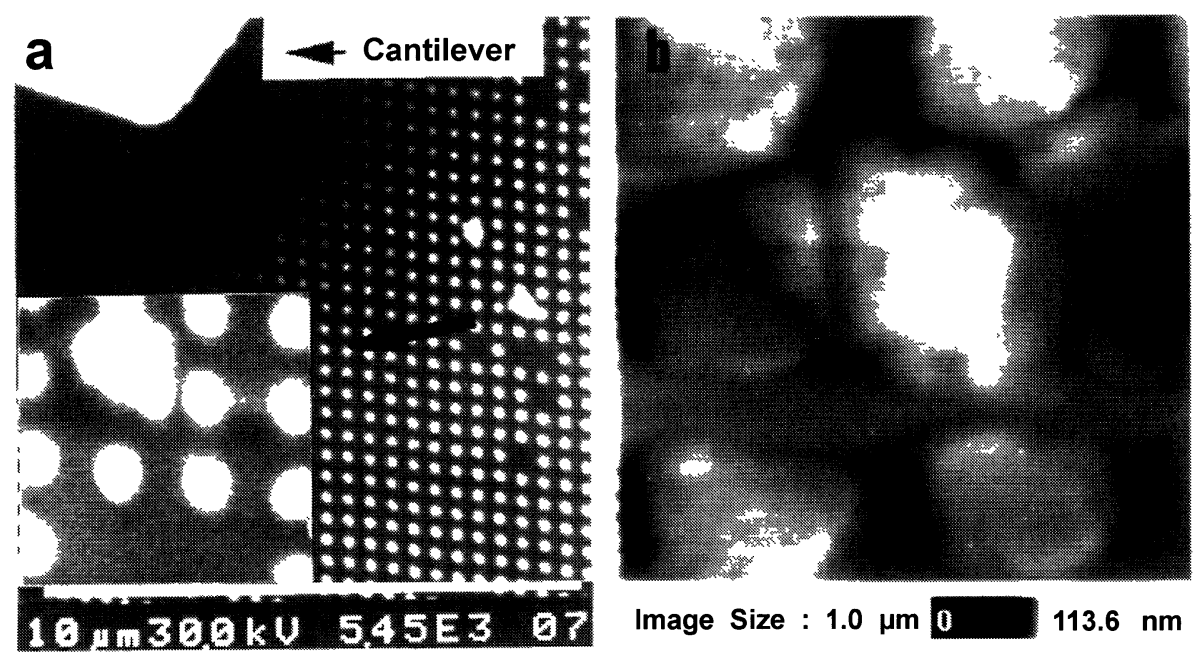

Fig. 5. - SFM/SEM images of a grating. a) SEM image with the view of the cantilever and a small magnified defect; b) SFM image of the same small defect. The studs appear as squares. This is an artefact due to the SFM tip geometry.

$40 \mathrm{~nm}$. The tip height $(4 \mu \mathrm{m})$ is not high enough to be visualised by SEM because it is hidden by the top of the cantilever. Unlike the case of the STM/SEM combination in the same configuration (tip $45^{\circ}$ tilted with respect to the beam axis), the tip extremity cannot be seen, which is a small drawback. Nevertheless, it is easy to position the SFM tip on a detail when the latter has been located by SEM and then to correlate the resulting images. Figure 5b is the SFM image of a defect present in the grating. It reveals small structures on the stud surface that were not visible by SEM. One can also see that the studs appear as squares whereas they are circular in the SEM image. This is an artefact due to the tip shape. Taking into account the stud height $(80 \mathrm{~nm})$, the sides of the tip (which has a pyramidal shape) have clearly been in contact with the studs. The image combines the information coming from the object and from the tip. This is the well-known "convolution" effect (the exact term is "dilation" in mathematical morphology [4]) between specimen and tip due to the finite dimensions of the latter. Observation by SFM alone of a relatively rough surface yields a wrong topographical interpretation and this example demonstrates all the interest and complementarity of using both techniques together; SFM is the privileged tool to reveal fine structures and to measure heights precisely, whereas SEM is well-adapted to give a reliable lateral representation of rough objects.

The tribology of surfaces at the submicrometric scale can also be studied with our system. In order to measure frictional forces we chose the fast scan direction perpendicular to the long direction of the cantilever. The cartography of the lateral forces and the topographic image are simultaneously recorded. For the moment, the information obtained is essentially qualitative but recent developments show that quantification at the nanometric scale of friction coefficients can be performed [15]. Figure 6a is the SEM image of a ceramic sample composed of two phases: $\mathrm{Al}_{2} \mathrm{O}_{3}$, that is not conducting, and TiN, which is only slightly conducting. This material, used as a bioceramic, behaves under friction differently from alumina. Figures $6 \mathrm{~b}$ and $6 \mathrm{c}$ are SFM images of the surface topography and frictional forces, respectively. The white areas in Figure 6c are characteristic of larger frictional forces. The information contained in 


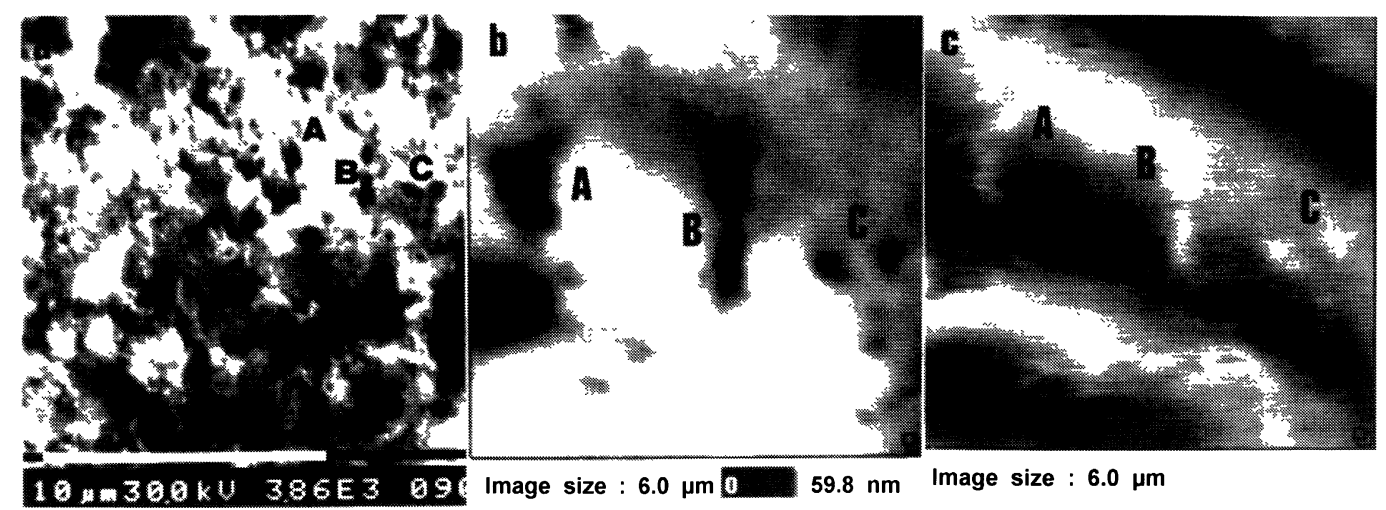

Fig. 6. - SFM/SEM images of an $\mathrm{Al}_{2} \mathrm{O}_{3}$ TiN bioceramic a) SEM image; b) topographical and c) frictional SFM images. Areas labelled with letters A, B, C correspond in the topography image to areas with small heights (dark colour), in the friction image to areas of high friction (light colour).

both SFM images in Figure 6 provides better understanding of the SEM image contrast. The SFM topographic image tells us that the dark spots in the SEM image are in fact at a lower height level and therefore one of the sample constituents may be preferentially pulled away by the mechanical polishing process. Some of these areas appear white in the frictional force image, showing that friction is more important there. Our SEM is not equipped with an X-ray analyser but X-ray cartography would certainly help to determine which phase has the lowest friction coefficient.

Our SFM is also able to give information about the viscoelastic properties of materials thanks to a working mode called "sample displacement modulation". The sample is periodically moved up and down ( $Z$ wobble) and the tip response is measured with a lock-in technique. The sample is displaced by means of a transducer placed underneath the sample (Fig. 1). We used a small piezo-electric disk of thickness $0.5 \mathrm{~mm}$. The frequency of the modulating signal must be chosen to be above the feedback response frequency (typically a few $\mathrm{kHz}$ ). There are two different working regimes: below the cantilever resonance frequency (at low frequencies $\sim 10 \mathrm{kHz}$ ) or well above it at high frequencies (between $\sim 100 \mathrm{kHz}$ and $1 \mathrm{MHz}$ ). The low frequency regime also called "the force modulation mode", is well-adapted to soft materials such as biological ones $[16,17]$. Measurement of the signal amplitude and phase gives the elasticity and viscosity response of the material, respectively [17]. The response of the cantilever to high frequency excitation is fundamentally different from its low frequency response. The theory of this regime has been developed by Burnham et al. [5]. Their analysis shows that modulating the position of the sample at frequencies above the highest system resonance gives the clearest difference in cantilever response for the variations in elastic modulus of stiff samples. The equations governing the cantilever behaviour at high frequencies being dominated by the acceleration, these authors named this working mode "Scanning Local-Acceleration Microscopy" (SLAM). Figure 7 is an example illustrating the capabilities of SLAM on a nickel-based superalloy single crystal. This material is composed of two phases $\gamma$ and $\gamma^{\prime}$, whose elastic moduli are very close, 130 and $115 \mathrm{GPa}$, respectively [9]. The $\gamma^{\prime}$ phase consists of cubic precipitates, which appear as dark squares in the topographic SFM image in Figure 7a since the single crystal was cut in slices with their main faces parallel to the (001) crystallographic plane [23]. The squares are slightly distorted because of hysteresis and non-linearity of the piezo-tube. After mechanical polishing (final step on $50 \mathrm{~nm}$ alumina powder), the $\gamma^{\prime}$ phase is revealed by dipping the sample 


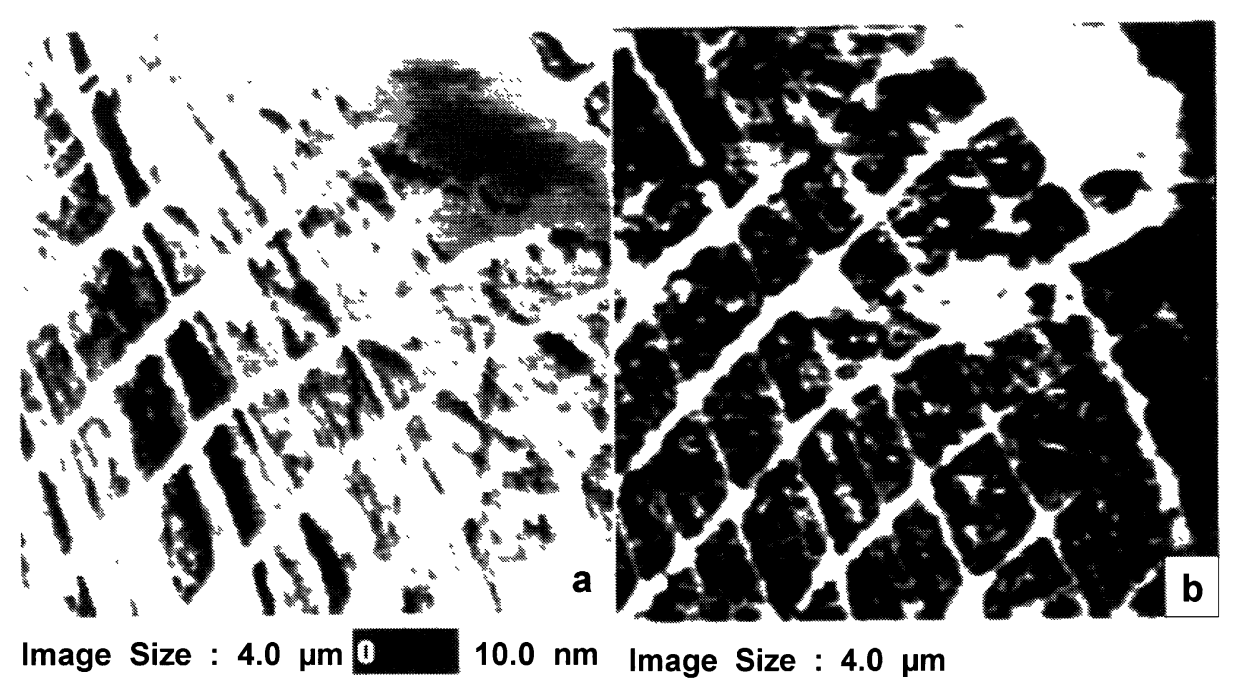

Fig. 7. - a) Topography and b) elasticity SFM images of a nickel based superalloy. The high frequency sample modulation displacement method is sufficiently sensitive to separate phases with close elastic moduli in a stiff material. The Young's moduli of the $\gamma^{\prime}$ phase (dark colour) and $\gamma$ phase (white colour) $\gamma$ are 115 and $130 \mathrm{GPa}$, respectively. The modulation signal amplitude is $z=2 \mathrm{~nm}$, frequency $f=188 \mathrm{kHz}$ and the cantilever stiffness $k=0,06 \mathrm{~N} / \mathrm{m}$.

for 15 seconds in a chemical solution that preferentially attacks the $\gamma^{\prime}$ phase. Therefore, the $\gamma$ phase (the matrix) appears in white (the highest height level on the sample) and the $\gamma^{\prime}$ phase in black (the lowest level). The SEM image is not presented here because the $\gamma^{\prime}$ precipitate depths are so small, between 5 and $10 \mathrm{~nm}$ as revealed by the topographic SFM image, that there is absolutely no contrast in the image. Figure $7 \mathrm{~b}$ is the SLAM image obtained from the amplitude response of the cantilever, with a modulation signal amplitude $z=2 \mathrm{~nm}$, frequency $f=188 \mathrm{kHz}$ and with a cantilever spring stiffness $k=0.06 \mathrm{~N} / \mathrm{m}$. The white areas in the image correspond to higher stiffness. The image shows clearly that the $\gamma$ phase has a higher stiffness than the $\gamma^{\prime}$ phase. The latter does not appear completely homogeneous; some small white spots are present inside. These, which correspond to a higher stiffness, are presumably small $\gamma$ particles pulled out during mechanical polishing, spread on the surface, still adhering to it and not removed during the short chemical etching. The high frequency sample displacement modulation method is so sensitive that "semi-quantitative" measurements of elastic moduli of stiff samples could be envisaged [13], semi-quantitative being understood here as the possibility of measuring the elastic modulus of one of the constituent of a material relative to another constituent, the Young's modulus of which is known and used as a reference.

We would like also to mention that there may be an additional advantage in performing SFM measurements or studies inside the vacuum of a SEM: the water layer that covers the specimen in air is desorbed under vacuum and so there are no more capillary forces. Viscoelastic and adherence studies can therefore be performed quantitatively. All the studies using the non-contact resonant mode also take advantage of the low pressure because the $Q$ factor of the cantilever resonance is much higher. This has been demonstrated for a magnetic force microscope combined with a SEM [12]. 


\section{Conclusion}

A scanning force microscope combined with a scanning electron microscope has been developed that has the possibility that a well-defined spot in a sample of a few $\mathrm{mm}^{2}$ can be observed with the aid of an $X-Y$ sample positioner using stepper motors of the inchworm type. These two instruments are complementary, the advantages of one compensating for the drawbacks of the other. Image correlation allows one to avoid some misinterpretations, relative to artefacts caused by tip-surface interactions in SFM or to contrast understanding in the SEM images. The presence of a SEM reduces the time and effort required in placing the SFM probe in the region of interest in a sample. From the point of view of the SEM user, the resolution is considerably improved and profilometry and roughness measurements at the nanometre scale thus become possible inside the SEM, with the SFM. The combination of these two techniques in the same instrument opens the way towards the construction of a multidimensional data space corresponding to the same place on the sample surface. The SEM gives access to information coming from secondary and backscattered electrons and allows X-ray analysis to be performed whereas the SFM, besides 3-D morphological analysis, allows nanotribological investigations (friction, wear, adhesion) and studies of some physical properties (viscoelastic, electric and magnetic) to be made.

With the multifunctional approach described, we believe that one can get new insights into the nature of materials and that such a SFM/SEM combination should become a practical tool in materials science, biology and in the semiconductor industry.

\section{Acknowledgments}

We would like to thank Prof. H. Gaub for giving us the electronic circuit drawings and the image acquisition and processing software, Drs. A. Hazotte and S. Begin-Colin of École des Mines of Nancy for supplying the nickel based superalloy and bioceramic $\mathrm{Al}_{2} \mathrm{O}_{3}$ TiN samples, respectively and M.E. Cambril for providing the periodic test specimen.

\section{References}

[1] Asenjo A., Buendia A., Gomez-Rodriguez J.M. and Baro A.M., Scanning tunnelling microscopy/scanning electron microscopy combined instrument, J. Vac. Sci. Technol. B 12 (1994) 1658-1661.

[2] Bhushan B., Israelachvili J.N. and Landman U., Nanotribology: friction, wear and lubrication at the atomic scale, Nature 374 (1995) 607-616.

[3] Binnig G., Quate C.F. and Gerber C.H., Atomic force microscope, Phys. Rev. Lett. 56 (1986) 930-933.

[4] Bonnet N., Dongmo S., Vautrot P. and Troyon M., A mathematical approach to image formation and image restoration in scanning tunnelling and atomic force microscopies, Microsc. Microanal. Microstruct. 5 (1994) 477-487.

[5] Burnham N.A., Kulik A.J., Gremaud G., Galto P.J. and Oulevey F., Scanning localacceleration microscopy, J. Vac. Sci. Technol. B 14 (1996) 794-799.

[6] Creuzet F., Ryschenkow G. and Arribart H., A new tool for adhesion science: the atomic force microscope, J. Adhesion 40 (1992) 15-21. 
[7] Ermakov A.V. and Garfunkel E.L., A novel AFM/STM/SEM system, Rev. Sci. Instrum. 65 (1994) 2853-2854.

[8] Fuchs H. and Laschinski R., Surface investigations with a combined scanning electronscanning tunnelling microscope, Scanning 12 (1990) 126-132.

[9] Ganghoffer J.F., Hazotte A., Denis S. and Simon A., Finite element calculation of internal mismatch stresses in single crystal base superalloy, Script. Metal. Matter. 25 (1991) 24912496.

[10] Gerber Ch., Binnig G., Fuchs H., Marti O. and Rohrer H., Scanning tunnelling microscope combined with a scanning electron microscope, Rev. Sci. Instrum. 57 (1996) 221-224.

[11] Ichinokawa T., Miyazaki Y. and Koga Y., Scanning tunnelling microscope combined with scanning electron microscope, Ultramicroscopy 23 (1987) 115-118.

[12] Kikukawa A., Hosaka S., Honda Y. and Koyanagi H., Magnetic force microscope combined with a scanning electron microscope, J. Vac. Sci. Technol. A 11 (1993) 3092-3098.

[13] Wang Z., Ph.D. Thesis (1997), Développement d'un microscope à force atomique multifonctionnel associé à un microscope à balayage, Université de Reims, France.

[14] Martin Y. and Wickramasinghe H.K., Magnetic imaging by force microscopy with $100 \mathrm{~nm}$ resolution, Appl. Phys. Lett. 50 (1987) 1455-1457.

[15] Overney R. and Meyer E., Tribological investigations using friction force microscopy, MRS Bull. 18 (1993) 26-34.

[16] Overney R., Meyer E., Frommer J. and Güntherodt H.J., Force microscopy study of friction and elastic compliance of phase-separated organic thin films, Langmuir 10 (1994) 1281-1286.

[17] Radmacher M., Tillmann R.W. and Gaub H.E., Imaging viscoelasticity by force modulation with the atomic force microscope, Biophysical J. 64 (1993) 735-742.

[18] Radmacher M., Ph.D. Thesis (1993), Ludwig-Maximilians Universität München, München, Germany.

[19] Stahl U., Yuan C.W., De Lozanne A.L. and Tortonese M., Atomic force microscope using piezoresistive cantilevers and combined with a scanning electron microscope, Appl. Phys. Lett. 65 (1994) 2879-2880.

[20] Terris B.D., Stern J.E., Rugar D. and Mamin H.J., Localised charge force microscopy, $J$. Vac. Sci. Technol. A 8 (1990) 374-377.

[21] Topometrix, 5043 Betsy Ross Drive, Santa Clara, CA 95054.

[22] Troyon M., Lei H.N. and Bourhettar A., Integration of an STM in a SEM, Ultramicroscopy 42-44 (1992) 1564-1568.

[23] Troyon M., Hazotte A. and Bourhettar A., Modelling the AFM tip effect for quantitative analysis of precipitate volume fraction in nickel-based superalloys, Microsc. Microanal. Microstruct. 5 (1994) 489-499.

[24] Vasquez L., Bartolome A., Garcia R., Buendia A. and Baro A.M., Combination of a scanning tunnelling microscope with a scanning electron microscope, Rev. Sci. Instrum. 59 (1988) 1286-1289. 